\title{
Toxicity of betulin derivatives and in vitro effect on promastigotes and amastigotes of Leishmania infantum and $L$. donovani
}

\author{
León Wert ${ }^{1}$, Sami Alakurtti ${ }^{2,3}$, María Jesús Corral ${ }^{1}$, Sebastián Sánchez-Fortún ${ }^{4}$, Jari Yli-Kauhaluoma ${ }^{2}$ \\ and José María Alunda ${ }^{1}$
}

The toxicity and antileishmanial activity of 20 betulin derivatives were studied. The toxicity of betulin and synthesized compounds was determined using a bacterial test (Microtox) and two mammalian cell lines (CHO-K1 and J774). The antileishmanial activity of compounds $(50 \mu \mathrm{m})$ was examined in both the promastigote and intracellular amastigote stages of Leishmania infantum and $L$. donovani. No correlation was found among the toxicity tests. All the compounds showed significant antipromastigote activity. The antiproliferative capacity of derivatives was dependent on the parasite stage studied, and no substantial differences were found between Leishmania species. Betulin, 3,28-di-O-acetylbetulin and L-aspartyl amide of betulonic acid showed moderate activity against amastigotes. The highest inhibition of intracellular amastigote multiplication was achieved with a low micromolar concentration $\left(\mathrm{IC}_{50} \mathrm{ca} 9 \mu \mathrm{M}\right.$ ) of heterocyclic betulin derivative 3,28-di-O-acetyllup-13(18)ene with $\mathrm{N}$-ethyltriazolo moiety 16 , without significant toxicity for mammalian cells. These results point to the interest of this lead compound for further in vitro and in vivo tests.

The Journal of Antibiotics (2011) 64, 475-481; doi:10.1038/ja.2011.34; published online 27 April 2011

Keywords: amastigotes; betulin; betulinic acid; cytotoxicity; Leishmania; L. infantum; promastigotes

\section{INTRODUCTION}

Leishmaniases are a group of vector-borne parasitic diseases caused by different species of Leishmania and present in all inhabited continents. It is estimated that around 12 million people are currently infected; over 350 million live in risk areas and 2 million new cases occur every year. ${ }^{1}$ Leishmaniasis has a range of clinical presentations, from selfhealing dermal processes (L. major and L. tropica) to visceral infections that are fatal if left untreated ( $L$. donovani). The infection was traditionally linked to tropical and subtropical regions but nowadays distribution is more widespread, as it is a common infection in immunocompromised patients. ${ }^{2}$ Human migrations due to war and famine have expanded the area of sandfly vectors, and human-induced environmental modifications have either led to the infection being found in areas where it was not previously present ${ }^{3}$ or have exacerbated its presence in other areas. ${ }^{4}$ Apart from human cases, dog infections ( $L$. infantum and $L$. chagasi) are of primary concern, both for public health considerations (zoonotic infections) and for strictly veterinary reasons. Conservative estimates suggest that in the Mediterranean region, at least $5-7 \%$ of the total dog population is infected ${ }^{5}$ and that there are 'hot spots' with a canine prevalence of over $30 \%$.
Different methods are employed to limit the spread of leishmaniasis (for example, medicated dog collars, insecticides where allowed) but, for the most part, the main system of control of infected individuals (humans and dogs) continues to be chemotherapy. A large number of drugs have been used, but the therapeutics of leishmaniasis is hampered by the toxicity of some of the compounds, difficulties in administration in remote areas, the intraspecific variability of Leishmania drug sensitivities and the development of resistances, particularly in the case of $L$. donovani in India. ${ }^{6,7}$ Moreover, in many countries, the use of first-line drugs (antimonials and amphotericins) is precluded by their high price. ${ }^{8}$ Despite impressive advances in the understanding of the biology and molecular biology of Leishmania spp, these have not yielded any substantial improvements on chemotherapy; generally speaking, the compounds either already in use or undergoing clinical trials are incidental findings rather than the result of a directed research effort. ${ }^{9}$ In 2004, World Health Organization/ Tropical Diseases Research (TDR) indicated that liposomal amphotericin, paromomycin and miltefosine were the most promising drugs against Leishmania on the market (http://apps.who.int/tdr/svc/ diseases/leishmaniasis). These three drugs, together with antimonials in those areas where resistance has not developed, constitute the

\footnotetext{
${ }^{1}$ Department of Animal Health, Faculty of Veterinary Medicine, Universidad Complutense de Madrid, Madrid, Spain; ${ }^{2}$ Division of Pharmaceutical Chemistry, Faculty of Pharmacy, University of Helsinki, Helsinki, Finland; ${ }^{3}$ Technical Research Centre of Finland, VTT, Espoo, Finland and ${ }^{4}$ Department of Pharmacology and Toxicology, Faculty of Veterinary Medicine, Universidad Complutense de Madrid, Madrid, Spain

Correspondence: Professor JM Alunda, Department of Animal Health, Faculty of Veterinary Medicine, Universidad Complutense de Madrid, Avda. Puerta de Hierro s/n, 28040 Madrid, Spain. 
reference treatments for leishmaniasis. However, none of these compounds is new. Amphotericin B has been used for decades as an effective treatment against leishmaniasis (in addition to its fungicidal efficacy); its main drawback, related to its toxicity, is that it has to be administered slowly under hospital conditions. Miltefosine (hexadecylphosphocholine) was originally developed as an anticancer agent, whereas the aminoglycoside antibiotic paromomycin (aminosidine) has been used as an antimicrobial since the 1960s. The need to explore new treatments is therefore recognized as a leading priority for research on leishmaniasis. ${ }^{10-13}$ Betulin (lup-20(29)-ene-3 $\beta, 28$-diol) is a pentacyclic triterpene widely distributed in nature. The outer bark of the birch (Betula sp) is particularly rich in this substance, which, depending on the species, may represent more than $25 \%$ of the bark by weight. This compound can be transformed into betulinic acid either by chemical transformation or biotransformation. ${ }^{14,15}$ It has been suggested that betulinic acid has anticancer, anti-HIV and antimicrobial properties. ${ }^{16,17}$ Some related compounds have shown antileishmanial activity against $L$. donovani (promastigotes and amastigotes) ${ }^{18}$ and L. amazonensis. ${ }^{19}$ More recently, studies have been carried out on the effect of a high number of betulinic derivatives on axenic amastigotes and amastigotes of $L$. donovani in the THP-1 cell line. ${ }^{20}$ It was found that the compound with greater antileishmanial capacity (heterocycloadduct between 3.28-di-O-acetyllupa-12,18diene and 4-methylurazine) showed a $50 \%$ growth inhibition $\left(\mathrm{IC}_{50}\right)$ of $8.9 \mu \mathrm{M}$ against amastigotes, although the compound showed a $30 \%$ toxicity for the THP-1 macrophage line. This toxicity possibly rules out its potential therapeutic use. In axenic amastigotes, the same group ${ }^{21}$ observed that betulonic acid exhibited the highest antileishmanial activity. L. infantum and L. donovani are closely related species. However, there are epidemiological and pathological differences between the infections caused by each agent. Moreover, given the inconsistencies of many drug-screening reports (the differential effect on promastigotes and amastigotes; the relative value of axenic amastigotes), it has been considered to be of interest to test an available series of betulin derivatives against promastigotes and amastigotes of L. infantum cultured in J774 macrophages.

\section{MATERIALS AND METHODS}

\section{Parasites}

Autochthonous isolates of L. infantum (UCM 6 and 10), obtained from affected dogs in the area of Madrid (Spain) by the Clinical Service of the Department of Animal Health of the Faculty of Veterinary Medicine (UCM), and 'Khartoum 1246 ' isolate from $L$. donovani, provided by Dr Toraño (Department of Immunology, Instituto de Salud Carlos III, Madrid), were routinely maintained as promastigotes in RPMI 1640 medium (Lonza Group, Switzerland) at $26^{\circ} \mathrm{C}$ supplemented with heat-inactivated $\left(30 \mathrm{~min}\right.$ at $\left.56^{\circ} \mathrm{C}\right)$ fetal bovine serum (Sera Laboratories International, Horsted Keynes, UK) and $100 \mathrm{U} \mathrm{ml}^{-1}$ penicillin $+100 \mu \mathrm{g} \mathrm{ml}^{-1}$ streptomycin (BioWhittaker, Verviers, Belgium) in 25-ml culture flasks.

\section{Macrophages}

J774 cells (murine monocyte-like cell line) were grown in RPMI 1640 medium in 25-ml flasks supplemented with fetal bovine serum and antibiotics, as above, in a humidified $5 \% \mathrm{CO}_{2} /$ air atmosphere at $37^{\circ} \mathrm{C}$.

\section{Compounds tested}

The 20 betulin derivatives tested in the present study were synthesized in the Division of Pharmaceutical Chemistry, Faculty of Pharmacy, University of Helsinki, Finland (Table 1). The chemical synthesis and detailed characterization data of the betulin derivatives are reported in our previous publications. ${ }^{15,20,22}$ Lyophilized compounds were resuspended in dimethyl sulfoxide for a 10-mu stock solution. The concentration of dimethyl sulfoxide in the working solution was no greater than $0.5 \%$ in any experiment. A standard concentration of $50 \mu \mathrm{M}$ was used in the first screenings after Chowdhury et al. ${ }^{18}$ Approximate $\mathrm{IC}_{50}$ of compound 16 was estimated with a range of concentrations $(0,10,25$ and $50 \mu \mathrm{M})$.

\section{Promastigote assay}

Promastigotes $\left(10^{6}\right.$ parasites $\left.\mathrm{ml}^{-1}\right)$ were cultured in $10-\mathrm{ml}$ sterile polystyrene tubes. Standard dilution of the compound $(50 \mu \mathrm{m})$ was added up to $2 \mathrm{ml}$ final volume. After $72 \mathrm{~h}$ at $26^{\circ} \mathrm{C}$, live promastigotes were counted in an improved Neubauer chamber (Marienfeld, Lauda-Königshofen, Germany). Results of growth inhibition were expressed as percentage (\%) of growth in untreated control cultures. Cultures were performed at least in triplicate, and amphotericin B was included as control.

\section{Amastigote assay}

In the amastigote assay, infection was carried out using a modification of the method developed by us. ${ }^{23}$ Briefly, $5 \times 10^{3} \mathrm{~J} 774$ cells per well were cultured in 16-well Lab-Tek chambers (Nunc, Roskilde, Denmark) for 16h. Stationary phase Leishmania promastigotes (days 8-11) were centrifuged without brake $(660 \times g, 10 \mathrm{~min})$ through a Ficoll gradient $(0,10$ and $20 \%)$ in fresh RPMI medium. Parasites recovered from the top were washed with fresh medium and opsonized with $5 \%$ normal mouse serum in a solution of $1: 1(\mathrm{v} / \mathrm{v})$ RPMI medium and HBSS $0.15 \mathrm{mM} \mathrm{CaCl}_{2}$ and $1 \mathrm{~mm} \mathrm{MgCl}_{2}$ for $30 \mathrm{~min}$. The washed parasites were added to the macrophage cultures (parasite: macrophage ratio 10:1) and maintained at $33^{\circ} \mathrm{C}$ in $5 \% \mathrm{CO}_{2}$ overnight. Non-internalized promastigotes were eliminated by washing three times with fresh medium. Compounds were added $(50 \mu \mathrm{M})$ and maintained for $48 \mathrm{~h}$ for preliminary screenings or for $72 \mathrm{~h}$ in subsequent determinations. Unless otherwise stated, mitomycin C (Calbiochem, Merck, USA) at $1 \mu \mathrm{g} \mathrm{ml}^{-1}$ was added at each stage to avoid host cell proliferation. Slides were fixed and stained (Diff-Quick, Dade Behring, Switzerland), and the number of amastigotes per 100 cells and percentage of infected cells were determined. Cultures were performed at least in triplicate, and activity controls with Fungizone (deoxycholate-dispersed amphotericin B, Bristol-Myers Squibb, Rueil-Malmaison, France; $0.2 \mu \mathrm{g} \mathrm{ml}^{-1}$ amphotericin B base) were included.

\section{Cell cytotoxicity assays}

Alamar blue reduction (J774 cells). J774 cells were counted in an improved Neubauer chamber (using trypan blue vital staining) and $1.25 \times 10^{4}$ cells per well in $200 \mu \mathrm{l}$ were placed in a 96-well plate with the standard dilution of the compound $(50 \mu \mathrm{M})$. After $48 \mathrm{~h}$, the medium was eliminated and $200 \mu \mathrm{l}$ of fresh supplemented medium plus $22 \mu \mathrm{l}$ of Alamar Blue reagent (Serotec, Oxford, UK) were added. Absorbance $(570$ and $600 \mathrm{~nm}$ ) was measured to calculate growth inhibition $\left(\% ; \mathrm{IC}_{50}\right) \cdot{ }^{24,25}$ Negative control cultures without compound were included. Cultures were performed in triplicate.

Neutral red assay (CHO-K1 cells). Cytotoxicity was estimated in CHO-K1 cells using the neutral red (3-amino-7-dimethylamino-2-methylphenazine hydrochloride) assay. ${ }^{26}$ This assay measures cellular transport based on the dye uptake by living cells. The absorbance change is directly proportional to the number of viable cells. For this analysis, $2 \times 10^{3}$ cells per well were cultured in 96-well plates and grown at $37^{\circ} \mathrm{C}$ in a $5 \% \mathrm{CO}_{2}$ humid atmosphere in a complete culture medium for $4 \mathrm{~h}$. This medium was then replaced with betulin derivatives. After $24 \mathrm{~h}$, the medium was removed and fresh medium containing $40 \mu \mathrm{g} \mathrm{ml}^{-1} \mathrm{RN}$ dye (Sigma, St Louis, MO, USA) was added. After a $3 \mathrm{~h}$ incubation, cells were washed with phosphate-buffered saline ( $\mathrm{pH}$ 7.2). Color was developed by the addition of $0.1 \mathrm{ml} 1 \%$ acetic acid in $50 \%$ ethanol. The plate was shaken for $10 \mathrm{~min}$, and absorbance was measured at $540 \mathrm{~nm}$ using an automatic FluoStar OPTIMA plate reader (BMG Labtech GmbH, Offenburg, Germany). Ethanol (5\%) was used as a positive control. The cytotoxicity percentage was calculated as $((\mathrm{A}-\mathrm{B}) / \mathrm{A} \times 100)$, where $\mathrm{A}$ and $\mathrm{B}$ are the absorbance of control and treated cells, respectively.

Microtox toxicity test. The Microtox basic test was carried out as preliminary screening using the manufacturer's (Microbics, Carlsbad, CA, USA) protocol. A Microtox Model 500 Analyzer (AZUR Environmental, Carlsbad, CA, USA) was used to measure the luminosity from Vibrio fischeri after 15-min exposure 
Table 1 Structures of betulin 1, semisynthetic betulin derivatives 2-15 and heterocyclic betulin derivatives 16-20

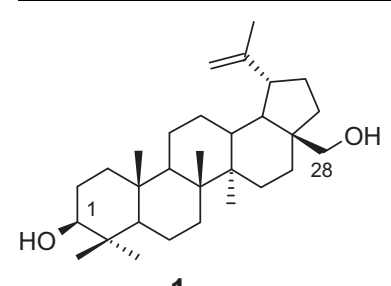

1

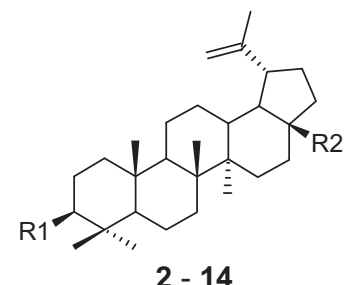

R1

$\mathrm{OH}$

$\mathrm{OH}$

$\mathrm{OH}$

$\mathrm{OH}$

$O A C$

$\mathrm{OAC}$<smiles>COC(=O)CCC(C)=O</smiles>

$\mathrm{OH}$

$\mathrm{OH}$

$\mathrm{O}=$

$0=$

$0=$

$\mathrm{OH}$

$=\mathrm{NOH}$



15

R2

$\mathrm{CH}_{2} \mathrm{OH}$<smiles>CCOC(=O)/C=C/c1ccccc1</smiles><smiles>CCOC(=O)c1cccnc1</smiles><smiles>CCOC(=O)CBr</smiles>

$\mathrm{OH}$

$\mathrm{CH}_{2} \mathrm{OAC}$<smiles>CCOC(=O)CCC(C)=O</smiles><smiles>C[C](C)COC1CCCCO1</smiles>

$\mathrm{CO}_{2} \mathrm{H}$

$\mathrm{CHO}$

$\mathrm{CO}_{2} \mathrm{H}$<smiles>COC(=O)C[C@H](NC(=O)[C@H](C)OC)C(=O)O</smiles>

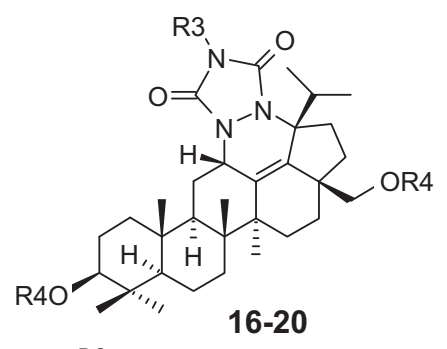

Compound

R3

R4

$\mathrm{Me}$

COEt

COi-Pr

to the tested compounds. Inhibition of light emission was measured in relative units of luminescence, and these data were used to calculate the median effective concentration $\left(\mathrm{IC}_{50}\right)$.
Statistical analysis

Results obtained within each species were analyzed by one-way analysis of variance test, and multiple comparisons were made using $P$-values corrected by 
the Bonferroni method. To compare the results obtained between Leishmania species, the Student's $t$-test was performed. In both cases, the level of significance was $P<0.05$. Statistical analysis was carried out with Statgraphics Centurion, Timberlabe Cons. Ltd, London, UK. Unless otherwise stated, all experiments were performed in triplicate.

\section{RESULTS}

\section{Effect of betulin derivatives on promastigote stage}

All the compounds tested were able to significantly reduce the multiplication of both parasite species (Table 2). In the series of simple betulin derivatives (compounds 2-15) where modifications were done to hydroxyl groups at C-3 and C-28, only few derivatives showed improved activity when compared with betulin 1 . In the subgroup of betulin esters (2-7), only 28-O-cinnamoylbetulin 2 showed improved activity at $50 \%$ against both parasite species. In addition, 28-O-nicotinoylbetulin 3 was more effective against $L$. infantum and 3-O-acetylbetulin 5 against $L$. donovani, respectively. The rest of the ester derivatives showed weak-to-moderate activity, with inhibition between 20 and $40 \%$. Ether derivative 28-O-tetrahydropyranylbetulin $\mathbf{8}$ was slightly less active than betulin 1. Oxidation of hydroxyl groups of betulin 1 had also only minor effects on antileishmanial activities (compounds 9-11). Betulonic aldehyde 10 and betulonic acid 11 with a ketone carbonyl as a functional group at C-3 were less active, and only betulinic acid 9 showed improved activity against L. donovani, but activity was not improved against $L$. infantum. L-aspartyl amide of betulonic acid 12 showed decreased activity against $L$. donovani. Betulin-28-oxime 13 was only weakly active, whereas betulin-3,28dioxime 14 with two oxime groups, at C-3 and C-28, showed improved activity against $L$. donovani. The 28-oxoallobetulone $\mathbf{1 5}$ showed decreased activity against $L$. infantum, whereas activity against $L$. donovani remained unchanged. In general, there can been seen a suggestive trend that betulin derivatives with free hydroxyl group at

Table 2 Effect of betulin derivatives $(50 \mu \mathrm{m})$ on the in vitro multiplication of Leishmania infantum and $L$. donovani promastigotes

\begin{tabular}{lll}
\hline Compound $^{\mathrm{a}}$ & L. infantum promastigotes & L. donovani promastigotes \\
\hline $\mathbf{1}$ & $42.8 \pm 12.7$ & $39.2 \pm 11.5$ \\
$\mathbf{2}$ & $53.3 \pm 6.4$ & $50.1 \pm 8.9$ \\
$\mathbf{3}$ & $53.8 \pm 12.0$ & $34.3 \pm 3.3$ \\
$\mathbf{4}$ & $39.5 \pm 6.9$ & $26.4 \pm 5.3$ \\
$\mathbf{5}$ & $32.9 \pm 8.9$ & $52.8 \pm 5.1$ \\
$\mathbf{6}$ & $21.5 \pm 12.1$ & $25.4 \pm 5.3$ \\
$\mathbf{7}$ & $35.3 \pm 14.9$ & $37.5 \pm 14.1$ \\
$\mathbf{8}$ & $36.4 \pm 4.0$ & $25.4 \pm 0.9$ \\
$\mathbf{9}$ & $36.0 \pm 11.7$ & $63.7 \pm 10.2$ \\
$\mathbf{1 0}$ & $26.5 \pm 6.8$ & $32.7 \pm 14.0$ \\
$\mathbf{1 1}$ & $30.6 \pm 9.2$ & $21.2 \pm 7.0$ \\
$\mathbf{1 2}$ & $42.9 \pm 4.3^{\mathrm{b}}$ & $12.6 \pm 13.0^{\mathrm{b}}$ \\
$\mathbf{1 3}$ & $22.1 \pm 2.1$ & $17.6 \pm 3.9$ \\
$\mathbf{1 4}$ & $33.8 \pm 12.8$ & $50.1 \pm 6.4$ \\
$\mathbf{1 5}$ & $24.0 \pm 3.2$ & $42.0 \pm 20.5$ \\
$\mathbf{1 6}$ & $25.5 \pm 9.9$ & $48.3 \pm 11.5$ \\
$\mathbf{1 7}$ & $30.8 \pm 6.0$ & $24.7 \pm 3.1$ \\
$\mathbf{1 8}$ & $18.4 \pm 4.4$ & $31.0 \pm 9.3$ \\
$\mathbf{1 9}$ & $63.8 \pm 4.3$ & $33.8 \pm 16.7$ \\
$\mathbf{2 0}$ & $34.6 \pm 7.0^{\mathrm{b}}$ & $20.9 \pm 1.6^{\mathrm{b}}$ \\
\hline 25 & &
\end{tabular}

Results (average \pm s.d.) represent the percentage of growth of untreated control cultures. aNumbering of compounds as in Table 1 .

${ }^{b}$ Statistically significant difference $(P<0.05)$ in the inhibitory effect on promastigotes from both species.
C-3 showed same or slightly improved activities against $L$. infantum (except for betulin-28-oxime 13), whereas modification at C-3 decreased the activity. In the case of $L$. donovani, no such trend can be seen, although compounds with ketone carbonyl at C-3 (10-13) were less active than betulin 1 .

In the subgroup of heterocyclic betulin derivatives (16-20), clearer structure-activity relationship trend could be observed. Compounds with sterically less bulky R3 and R4 groups showed better activity, when compared with more hindered derivatives. Heterocycloadduct $\mathbf{1 6}$ with ethyl as an R3 group and acyl moieties as R4 groups showed better activity than $\mathbf{1 7}$ or 18, which have bulky aromatic R3 groups. When comparing cycloadducts 19 with $\mathbf{2 0}$, both of which have methyl as an R3 group, derivative having less bulky R4 was more active. Overall, antileishmanial activity of betulin derivatives was depending on the studied parasite species. Some derivatives that showed good activity against $L$. infantum were only moderate or weakly active against $L$. donovani promastigotes and vice versa. Approximate $\mathrm{IC}_{50}$ value of amphotericin, included as positive control compound, was $0.07 \mu \mathrm{m}$ for both species after $48 \mathrm{~h}$ incubation.

\section{Toxicity of betulin derivatives}

Toxicity of the compounds for $V$. fischeri and two mammalian cell lines (CHO-K1 and J774) was examined. In addition, a qualitative microscopic evaluation was performed using J774 cells. Differences in toxicity were found among the betulin derivatives tested, and no correlation was observed between the three methods employed. The Microtox test displayed the highest variation of inhibition ranging from 0 to $100 \%$ (Table 3 ). When CHO-K1 cells were exposed to the betulin-derived compounds assayed $(50 \mu \mathrm{M})$, all the compounds except 18 were lacking in toxicity, with inhibition values below $10 \%$.

Table 3 Toxic effects induced by selected betulin derivatives on Microtox test and mammalian cell (CHO-K1 and J774 cell lines) toxicity assays

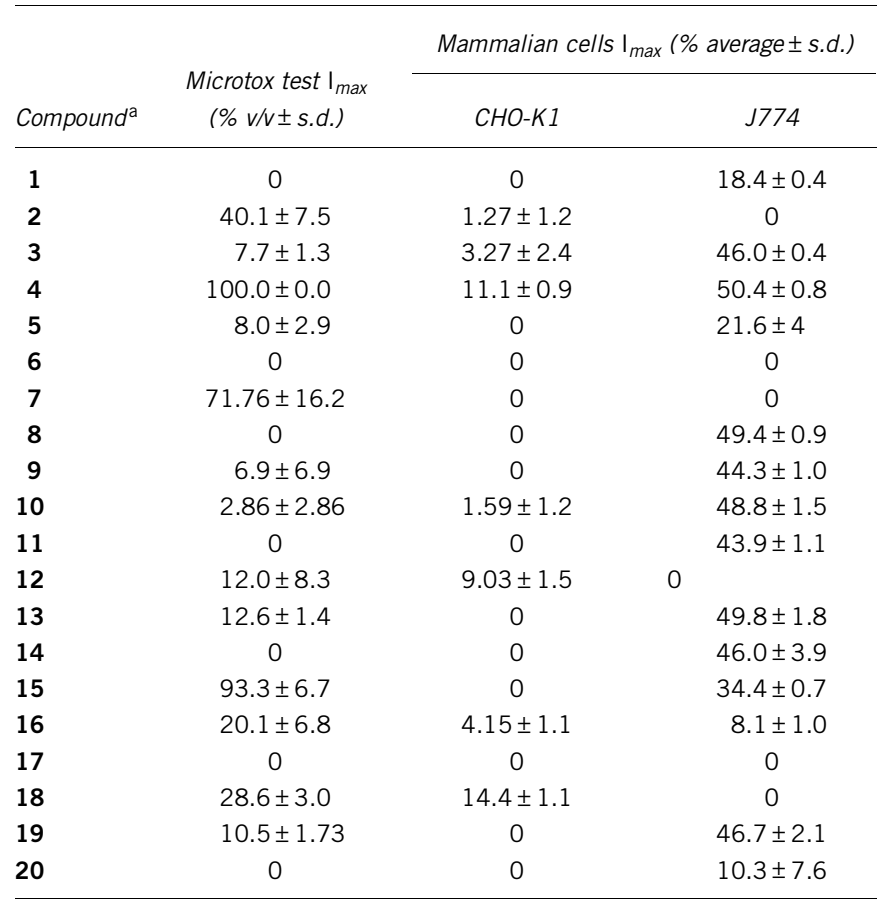

Values are shown as maximal inhibition $\left(I_{\max }\right)$ percentages \pm s.d. with respect to untreated controls.

aNumbering of compounds as in Table 1. 


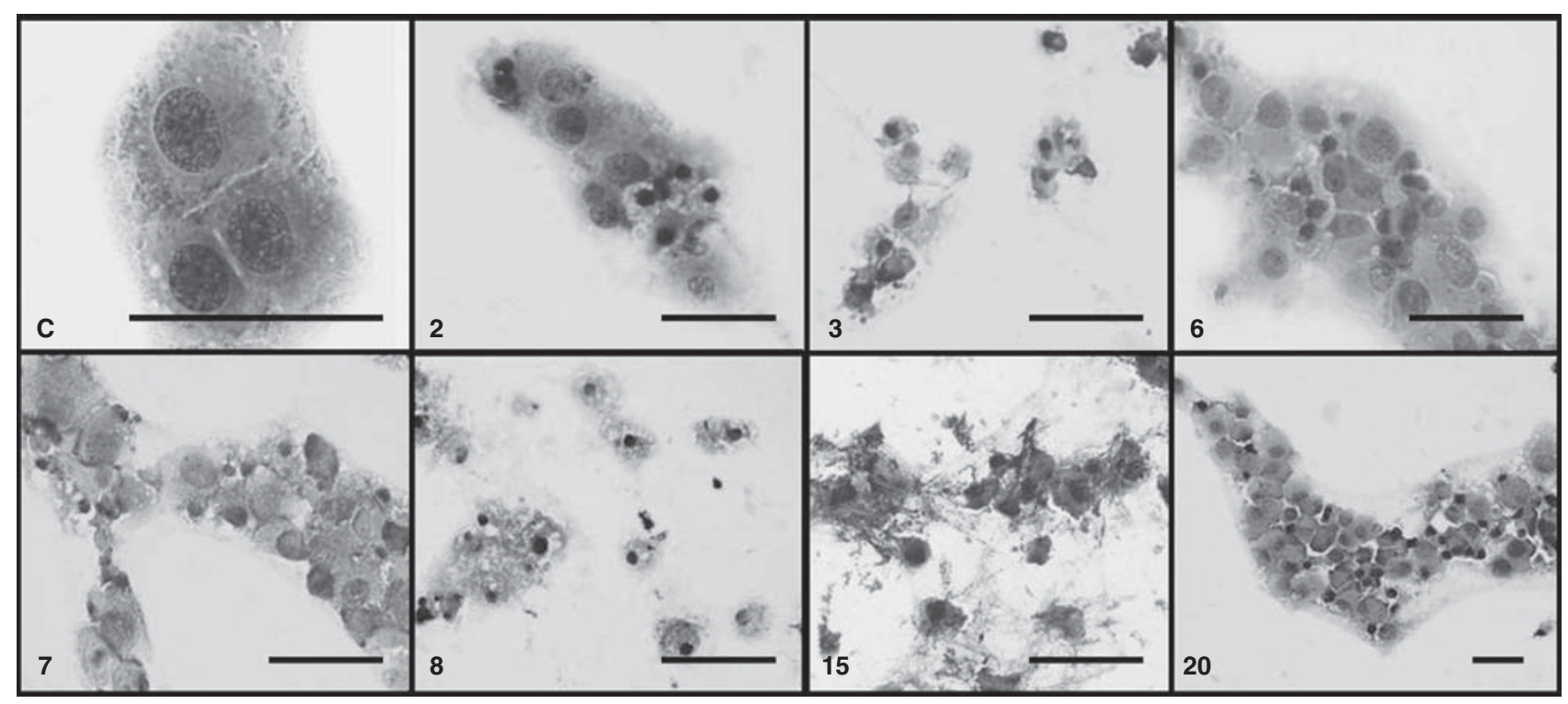

Figure 1 Representative microscopic fields of the in vitro effect of some betulin derivatives $(50 \mu \mathrm{m})$ on the Diff-Quick-stained murine macrophage cell line J774 after 72-h exposition. C, untreated control culture. Numbers correspond to those in Table 1. Bar: $50 \mu \mathrm{m}$. A full color version of this figure is available at The Journal of Antibiotics journal online.

However, when the compounds were added to the macrophage murine line J774 and cell growth inhibition was determined by the Alamar Blue colorimetric method, the inhibitory effect was found to be much higher; after $48 \mathrm{~h}$ of coincubation with the betulin derivatives, nine compounds actually showed reductions of over $40 \%$ when compared with the untreated control cultures. Some compounds did not show any noticeable toxicity with this method $(2,7,16,17$ and 20), and no apparent structure-activity relationship was observed between toxicity for the J774 cells and the compound subgroup (that is, derivatives having C-3 ketone carbonyl: compounds $\mathbf{1 0}$ and $\mathbf{1 1}$ were toxic, but L-aspartyl amide of betulinic acid 12 was non-toxic). However, all betulin derivatives having both hydroxyl groups acetylated $(6,16,17,18)$ were non-toxic to J774 cells. It is noticeable that even betulin 1 exhibited certain toxicity ( $18.4 \pm 0.4 \%$ inhibition). Moreover, the effect was observed both with mitomycin and without the multiplication inhibitor. Toxicity for the macrophage was further assessed by microscopic examination. Unfortunately, only eight compounds could be used. However, in the cases examined, a good correlation was found between the Alamar Blue results and microscopy in J774 cells. Figure 1 shows the effect of some derivatives, with toxicity estimated by the colorimetric method ranging between $40-50 \%$ (3, 4 and 15), as compared with the scarce effect on cell integrity and staining pattern of non-toxic compounds (2, 7, 8 and 20).

\section{Effect of betulin derivatives on Leishmania amastigotes in macrophages}

Only compounds that showed an inhibitory effect on the mammalian cell line $\mathrm{J} 774 \leqslant 20 \%$ were extensively tested. All the compounds assayed were able to reduce the multiplication of intracellular amastigotes of Leishmania as compared with the uninfected control cultures (Table 4). In the subgroup of simple betulin derivatives (1-15), only four compounds $(1,2,6$ and 12) were tested against intracellular $L$. infantum and $L$. donovani amastigotes. Betulin $\mathbf{1}$ showed best activity ( 49.3 and $42.8 \%$, respectively), whereas rest of the compounds showed similar or decreased activity.

When comparing intracellular amastigotes inhibition with promastigote inhibition activity, betulin $\mathbf{1}$ showed slightly improved activity
Table 4 Effect of some betulin derivatives $(50 \mu \mathrm{m})$ on amastigotes of Leishmania infantum and $L$. donovani cultured in $\mathbf{J 7 7 4}$ macrophage cell line

\begin{tabular}{lcc}
\hline & \multicolumn{2}{c}{ Amastigote \% average \pm s.d. reduction } \\
\cline { 2 - 3 } Compound $^{\mathrm{a}}$ & L. infantum & L. donovani \\
\hline 1 & $49.3 \pm 9.0$ & $42.8 \pm 22.1$ \\
2 & $44.0 \pm 8.6$ & $29.5 \pm 6.3$ \\
3 & $\mathrm{ND}$ & $\mathrm{ND}$ \\
4 & $\mathrm{ND}$ & $\mathrm{ND}$ \\
5 & $\mathrm{ND}$ & $\mathrm{ND}$ \\
6 & $25.5 \pm 18.0$ & $43.1 \pm 11.7$ \\
7 & $\mathrm{ND}$ & $\mathrm{ND}$ \\
8 & $\mathrm{ND}$ & $\mathrm{ND}$ \\
9 & $\mathrm{ND}$ & $\mathrm{ND}$ \\
10 & $\mathrm{ND}$ & $\mathrm{ND}$ \\
11 & $\mathrm{ND}$ & $\mathrm{ND}$ \\
12 & $44.9 \pm 11.6$ & $30.0 \pm 3.8$ \\
13 & $\mathrm{ND}$ & $\mathrm{ND}$ \\
14 & $\mathrm{ND}$ & $\mathrm{ND}$ \\
15 & $\mathrm{ND}$ & $\mathrm{ND}$ \\
16 & $70.4 \pm 13.5$ & $75.9 \pm 5.8$ \\
17 & $51.2 \pm 5.5$ & $20.8 \pm 20.6$ \\
18 & $40.7 \pm 12.1$ & $14.7 \pm 9.7$ \\
19 & $\mathrm{ND}$ & $\mathrm{ND}$ \\
20 & $\mathrm{ND}$ & $\mathrm{ND}$ \\
\hline
\end{tabular}

Abbreviation: ND, not determined.

Values are means \pm s.d. reduction (\%) of untreated control cultures.

aNumbering of compounds as in Table 1.

against both $L$. infantum and $L$. donovani amastigotes, with 49.3 and $42.8 \%$ inhibition, respectively. Also, moderately active 3,28-di-Oacetylbetulin 6 and L-aspartyl amide of betulonic acid 12 were more active against amastigotes than promastigotes. On the other hand, 28$O$-cinnamoylbetulin 2 was less active against amastigotes. From the subgroup of heterocyclic betulin derivatives (16-20), compounds 16, 
17 and 18 were screened against Leishmania amastigotes growing in macrophages. The activity of sterically less hindered cycloadduct $\mathbf{1 6}$ (over $70 \%$ inhibition against both $L$. infantum and $L$. donovani amastigotes, R3=Et) was clearly better than that of cycloadduct 17 $(\mathrm{R} 3=\mathrm{Ph})$ or $18(\mathrm{R} 3=$ indan-5-yl). The exploration of different concentrations of the most active compound (16) yielded an $\sim 50 \% \mathrm{IC}_{50}$ of $9 \mu \mathrm{M}$ (not shown). Under our conditions, $\mathrm{IC}_{90}$ was not achieved in the concentration range employed $(0-50 \mu \mathrm{M})$. Amphotericin B showed an $\mathrm{IC}_{50}$ value of $0.09-0.1 \mu \mathrm{m}$ for both Leishmania species.

\section{DISCUSSION}

Our results showed that some of the betulin derivatives tested had higher antileishmanial activity than the base compound of the series, betulin 1 , against amastigotes of $L$. infantum and $L$. donovani in macrophages. Of these, derivative $\mathbf{1 2}$ was previously tested with macrophages infected with $L$. donovani amastigotes, ${ }^{21}$ and earlier results $(53 \%$ inhibition at $50 \mu \mathrm{M})$ were in agreement with the present results (69\% inhibition). Under our conditions, the highest inhibition activity against intracellular amastigotes of both Leishmania species was obtained with derivative $16, \mathrm{IC}_{50}$ value in the micromolar range (ca $9 \mu \mathrm{M}$ ). Better antileishmanial efficiency of betulin heterocycloadducts with less bulky $\mathrm{N}$ - and $\mathrm{O}$-substituents is in accordance with our earlier results with $L$. donovani axenic amastigotes (strain MHOM/SD/1962/ 1S-Cl2d; $\left.\mathrm{IC}_{50} 30 \mu \mathrm{M}\right)$ and intracellular amastigotes in THP-1 macrophages, where this compound showed $53 \%$ inhibition at $25 \mu \mathrm{m} .{ }^{20}$

The activity found with $\mathbf{1 6}$ against intracellular Leishmania, without substantial toxicity for host cells, points to the interest of carrying out further tests of this compound on animal models. The mechanism of action of betulin and betulin derivatives has been only partially explored (it prevents DNA cleavage and the formation of topoisomerase-DNA complex), ${ }^{18,27-29}$ and internalization by Leishmania is unknown. It is interesting to note that the most active derivative exhibited a higher inhibitory activity against amastigotes than against promastigotes. This finding may indicate the presence of a host cellmediated mechanism and/or a mechanism involving different targets in hosts and parasite. This should be explored with the lead compound identified. The correlation found between the inhibition results against the amastigote from both Leishmania spp. was higher than that found between the amastigote and promastigote stages; results obtained in the intracellular stage in both species of Leishmania were comparable. This is consistent with the close intrahost life cycle and the similar sensitivities to well-established antileishmanial drugs (that is, amphotericin B).

Microscopic evaluation of toxicity against macrophages was as sensitive as the colorimetric method used (Alamar Blue), in spite of being labor and time consuming. The estimated toxicity of tested compounds was highly variable depending on the bioassay, even with those involving the use of mammalian cells (CHO-K1 and J774 cell lines). The $V$. fischeri bacteria test (Microtox) has been found to be correlated with other tests in higher organisms. ${ }^{30}$ However, in our case, no relationship was found.

Under our conditions, antileishmanial activity of betulin derivatives against the promastigote stage was dependent on the parasite species tested. Some derivatives that showed good activity against $L$. infantum were only moderate or weakly active against promastigotes of the $L$. donovani line used and vice versa. On the other hand, the lack of correlation between the results against promastigotes and amastigotes points to the need for using at least the intracellular stage in macrophages as the screening method to identify lead compounds against Leishmania. This need has been recognized, ${ }^{25,31,32}$ and is possibly critical for reducing the attrition rates of drug discovery.
Moreover, in our earlier study with $L$. donovani axenic amastigotes (strain MHOM/SD/1962/1S-Cl2d), derivatives 2 and $\mathbf{6}$ were totally inactive, ${ }^{21}$ whereas these compounds significantly inhibited the proliferation of amastigotes in macrophages. Thus, in any anti-Leishmania drug screening, infected macrophages should be preferred for identifying potentially useful drugs.

In conclusion, we found that all tested betulin derivatives showed inhibitory activity against $L$. donovani and $L$. infantum promastigotes. Toxicity for mammalian macrophages was variable and generally higher than that of betulin, which probably excludes the majority of these compounds as potential antileishmanial products. However, compound 16 strongly $(>70 \%)$ inhibited multiplication of the intracellular stages-those actually causing the disease in the infected hosts. Its activity against the two Leishmania species, and $\mathrm{IC}_{50}$ value in the micromolar range without significant toxicity for macrophages, points to the interest of this derivative. The pharmacology of this compound is not known, but structurally related betulinic acid, after i.p. injection, reached a peak serum concentration of $4 \mu \mathrm{g}$ (ca $6.01 \mu \mathrm{M}),{ }^{33}$ a value close to the estimated $\mathrm{IC}_{50}$ for $\mathbf{1 6}$. If bioavailability of the tested derivative were comparable, then $\mathbf{1 6}$ would be a promising lead for chemotherapy of leishmaniasis, which would suggest further studies of the disease in animal models using mice or hamsters.

\section{ACKNOWLEDGEMENTS}

This study was supported by the Spanish Ministry of Science and Innovation (CICYT-AGL2009-13009), the Finnish Funding Agency for Technology and Innovation (Tekes), the Foundation for Research of Natural Resources in Finland, Marjatta ja Eino Kollin Säätiö, the European Commission (contract no. EU-KBBE-227239-ForestSpeCs) and the COST Action CM-0801 (New drugs for neglected diseases). We thank Anja Salakari, Tuomo Heiska, Jukka Pernilä and Erkki Metsälä for their excellent technical assistance. We also thank Dr Salme Koskimies for her valuable discussions.

1 World Health Organization, WHO (who.int/health-topics/leishmaniasis.htm) (2000)

2 Desjeux, P. Leishmaniasis: current situation and new perspectives. Comp. Immunol. Microbiol. Infect. Dis. 27, 305-318 (2004).

3 Shaw, J. The leishmaniases-survival and expansion in a changing world. Mem. Inst. Oswaldo Cruz. 102, 541-547 (2007).

4 Reithinger, R. \& Coleman, P. G. Treating cutaneous leishmaniasis patients in Kabul, Afghanistan: cost-effectiveness of an operational program in a complex emergency setting. BMC Infect. Dis. 7, 3 (2007).

5 Morillas, F. et al. Leishmaniosis in the focus of the Axarquía region, Malaga province, southern Spain. Parasitol. Res. 82, 569-570 (1996).

6 Croft, S. L. \& Coombs, G. H. Leishmaniasis-current chemotherapy and recent advances in the search for novel drugs. Trends Parasitol. 19, 502-508 (2003).

7 Croft, S. L., Sundar, S. \& Fairlamb, A. H. Drug resistance in leishmaniasis. Clin. Microbiol. Rev. 19, 11-126 (2006).

8 Sundar, S. \& Chaterjee, M. Visceral leishmaniasis-current therapeutic modalities. Indian J. Med. Res. 123, 345-352 (2006).

9 Guerin, P. J. et al. Visceral leishmaniasis: current status of control, diagnosis, and treatment, and a proposed research and development agenda. Lancet Inf. Dis. 2, 494-501 (2002).

10 Alvar, J., Croft, S. L. \& Olliaro, P. Chemotherapy in the treatment and control of leishmaniasis. Adv. Parasitol. 61, 224-274 (2006).

11 Roberts, M. T. Current understandings on the immunology of leishmaniasis and recent developments in prevention and treatment. Br. Med. Bull. 75 \& 76, 115-130 (2006).

12 Chappuis, F. et al. Visceral leishmaniasis: what are the needs for diagnosis, treatment and control? Nat. Rev. Microbiol. 5, 873-882 (2007).

13 Mishra, J., Saxena, A. \& Singh, S. Chemotherapy of leishmaniasis: past, present and future. Curr. Med. Chem. 14, 1153-1169 (2007).

14 Alakurtti, S., Mäkelä, T., Koskimies, S. \& Yli-Kauhaluoma, J. Pharmacological properties of the ubiquitous natural product betulin. Eur. J. Pharm. Sci. 29, 1-13 (2006).

15 Pohjala, L., Alakurtti, S., Ahola, T., Yli-Kauhaluoma, J. \& Tammela, P. Betulin-derived compounds as inhibitors of alphavirus replication. J. Nat. Prod. 72, 1917-1926 (2009).

16 Ehrhardt, H., Fulda, S., Fuhrer, K. M., Debatin, K. M. \& Jeremias, I. Betulinic acidinduced apoptosis in leukemia cells. Leukemia 18, 1406-1412 (2004). 
17 Mukherjee, P. K., Saha, K., Das, J., Pal, M. \& Saha, B. P. Studies on the anti-inflammatory activity of rhizomes of Nelumbo nucifera. Planta Med. 63, 367-369 (1997).

18 Chowdhury, A. R. et al. Dihydrobetulinic acid induces apoptosis in Leishmania donovani by targeting DNA topoisomerase I and II: implications in antileishmanial therapy. Mol. Med. 9, 26-36 (2003).

19 Sauvain, M. et al. Isolation of leishmanicidal triterpenes and lignans from Amazonian liana Doliocarpus dentatus. Phytother. Res. 10, 1-4 (1996).

20 Alakurtti, S. et al. Synthesis and anti-leishmanial activity of heterocyclic betulin derivatives. Biorg. Med. Chem. 18, 1573-1582 (2010).

21 Alakurtti, S., Bergström, P., Sacerdoti-Sierra, N., Jaffe, Ch. F. \& Yli-Kauhaluoma, J. Anti-leishmanial activity of betulin derivatives. J. Antibiot. 63, 123-126 (2010).

22 Salin, O. et al. Inhibitory effect of the natural product betulin and its derivatives against the intracellular bacterium Chlamydia pneumoniae. Biochem. Pharmacol. 80, 1141-1151 (2010)

23 Méndez, S., Nell, M. \& Alunda, J. M. Leishmania infantum: infection of macrophages in vitro with promastigotes. Int. J. Parasitol. 26, 619-622 (1996).

24 Mikus, J. \& Steverding, D. A simple colorimetric method to screen drug cytotoxicity against Leishmania using the dye Alamar Blue. Parasitol. Int. 48, 265-269 (2000).

25 Ordóñez-Gutiérrez, L. et al. In vitro effect of new formulations of amphotericin B on amastigote and promastigote forms of Leishmania infantum. Int. J. Antimicrob. Agents 30, 325-329 (2007).
26 Borenfreud, E. \& Puerner, J. A. A simple quantitative procedure using monolayer culture for toxicity assays. J. Tissue Cult. Meth. 9, 7-9 (1984).

27 Pisha, E. et al. Discovery of betulinic acid as a selective inhibitor of human melanoma that functions by induction of apoptosis. Nat. Med. 1, 1046-1051 (1995).

28 Fulda, S. et al. Betulinic acid triggers CD95 (APO-1/Fas)- and p53-independent apoptosis via activation of caspases in neuroectodermal tumors. Cancer Res. 57, 4956-4964 (1997)

29 Chowdhury, A. R. et al. Betulinic acid, a potent inhibitor of eukaryotic topoisomerase I: identification of the inhibitory step, the major functional group responsible and development of more potent derivatives. Med. Sci. Monit. 8, BR254-BR265 (2002).

30 Kaiser, K. L. E. Correlations of Vibrio fischeri bacteria test data with bioassay data for other organisms. Environ. Health Perspect. 106(Suppl 2), 583-591 (1998).

31 Sereno, D., Cordeiro da Silva, A., Mathieu-Daude, F. \& Ouaissi, A. Advances and perspectives in Leishmania cell based drug-screening procedures. Parasitol. Int. 56, 3-7 (2007).

32 Vermeersch, M. et al. In vitro susceptibility of Leishmania donovani promastigote and amastigote stages to antileishmania reference drugs: practical relevance of stagespecific differences. Antimicrob. Agents Chemother. 53, 3855-3859 (2009).

33 Jaeger, S., Laszczyk, M. N. \& Scheffler, A. A preliminary pharmacokinetic study of betulin, the main pentacyclic triterpene from extract of outer bark of birch (Betula alba cortex). Molecules 13, 3224-3235 (2008). 